MATHEMATICS OF COMPUTATION

Volume 69, Number 230, Pages 501-520

S 0025-5718(99)01138-2

Article electronically published on March 18, 1999

\title{
THE CONVERGENCE \\ OF THE CASCADIC CONJUGATE-GRADIENT METHOD APPLIED TO ELLIPTIC PROBLEMS IN DOMAINS WITH RE-ENTRANT CORNERS
}

\author{
VLADIMIR SHAIDUROV AND LUTZ TOBISKA
}

\begin{abstract}
We study the convergence properties of the cascadic conjugategradient method (CCG-method), which can be considered as a multilevel method without coarse-grid correction. Nevertheless, the CCG-method converges with a rate that is independent of the number of unknowns and the number of grid levels. We prove this property for two-dimensional elliptic second-order Dirichlet problems in a polygonal domain with an interior angle greater than $\pi$. For piecewise linear finite elements we construct special nested triangulations that satisfy the conditions of a "triangulation of type $(h, \gamma, L)$ " in the sense of I. Babuška, R. B. Kellogg and J. Pitkäranta. In this way we can guarantee both the same order of accuracy in the energy norm of the discrete solution and the same convergence rate of the CCG-method as in the case of quasiuniform triangulations of a convex polygonal domain.
\end{abstract}

\section{INTRODUCTION}

In this paper, we consider a cascadic conjugate-gradient method (CCG-method) for solving discretized elliptic equations that yield discrete symmetric positive definite problems. This algorithm can be considered as a multigrid or multilevel method, but without coarse grid correction, i.e., if a certain grid level is attained, we do not return to coarser grid levels but proceed only at the same or on higher grid levels. The CCG-method can be recursively defined as follows. On the coarsest grid, the linear system is solved directly. On finer grids, the system is solved iteratively by the conjugate-gradient method. These iterations are started by an interpolation of the approximate solution from the previous coarser grid. On each fixed grid level we do not use any preconditioning based on coarser grids nor any restrictions onto coarser grid levels. Nevertheless, the CCG-algorithm as a multilevel method has optimal arithmetic complexity, and its convergence rate is independent of the number of unknowns and of the number of grid levels.

A CCG-algorithm has been recently presented by P. Deuflhard in [4] and [5], where the excellent convergence properties of this algorithm were demonstrated by numerical test examples. Its optimal arithmetic complexity with respect to the number of unknowns was proved for $H^{2}$-regular elliptic problems in [12]. Then for

Received by the editor November 11, 1997 and, in revised form, July 10, 1998.

1991 Mathematics Subject Classification. Primary 65F10; Secondary 65N30.

Key words and phrases. Multigrid, cascadic algorithm, conjugate-gradient method, finite element method.

The research was supported by the Deutsche Forschungsgemeinschaft. 
quasiuniform meshes this result was extended in [11] and 2] to elliptic problems with reduced regularity caused by interior angles greater than $\pi$. Nevertheless, the use of piecewise linear finite elements on quasiuniform triangulations reduces the convergence order of the Galerkin solution. Furthermore, F.A. Bornemann [2] studied the replacement of the CG-method by other iterative methods (damped Jacobi, Gauß-Seidel, SSOR, etc.) and gave sufficient conditions for optimal complexity of the cascadic algorithm. In the three-dimensional case these conditions are satisfied by many known iterative schemes, but in two dimensions the amount of work is suboptimal unless the CG-method is used.

Here we use piecewise linear finite elements on triangles in order to discretize a second-order elliptic problem in a polygonal domain with an interior angle greater than $\pi$. We construct special nested triangulations that are refined towards this angular point as in 13 and satisfy the conditions of a "triangulation of type $(h, \gamma, L)$ "; these were defined in 11 and used for the classical multigrid method in [14. We prove in detail that one obtains the same order of accuracy of the approximate solution and the same convergence rate of the CCG-method in the energy norm as in the $H^{2}$-regular case.

\section{The CASCADiC ALGORITHM}

We denote by $M_{0}, M_{1}, \cdots, M_{l}$ finite-dimensional vector spaces of increasing dimension equipped with inner products $(\cdot, \cdot)_{i}$, for $i=0,1, \cdots, l$. Moreover, let linear prolongation operators

$$
I_{i}: M_{i} \rightarrow M_{i+1}, \quad \text { for } i=0,1, \cdots, l-1,
$$

and linear invertible operators

$$
L_{i}: M_{i} \rightarrow M_{i}, \quad \text { for } i=0, \cdots, l,
$$

be given. Then the cascadic algorithm is an iterative method for solving the following problem:

For a given $f_{l} \in M_{l}$, find $u_{l} \in M_{l}$ such that

$$
L_{l} u_{l}=f_{l},
$$

by using approximations of the solutions of the following problems:

For a given $f_{i} \in M_{i}$, find $u_{i} \in M_{i}$ such that

$$
L_{i} u_{i}=f_{i}
$$

on lower levels $i=0, \cdots, l-1$. The idea is to start with the exact solution

$$
v_{0}=u_{0}
$$

on the lowest level $i=0$ and to prolong each approximate solution

$$
v_{i} \in M_{i}
$$

of (2.4) to the next higher level in order to find an initial guess for an iterative method that approximates the solution $u_{i+1}$. Applying the conjugate-gradient algorithm (CG-algoritm) on each level, we obtain the cascadic conjugate-gradient algorithm (CCG-algorithm), which can be formulated in the following way: 


\section{CCG-algorithm}

\{1. Set $v_{0}=L_{0}^{-1} f_{0}$.

2. For $i=1,2, \cdots, l$ and given $v_{i-1}$ do:

$\left\{\quad\right.$ 2.1. Set $w_{i}=I_{i-1} v_{i-1}$;

2.2. Perform $m_{i}$ iterations of the conjugate-gradient method :

$$
\begin{aligned}
& y_{0}=w_{i} ; \\
& p_{0}=r_{0}=f_{i}-L_{i} y_{0} \text {; } \\
& \sigma_{0}=\left(r_{0}, r_{0}\right)_{i} \text {; } \\
& \text { for } k=1,2, \cdots, m_{i} \text { do : } \\
& \left\{\alpha_{k-1}=\sigma_{k-1} /\left(p_{k-1}, L_{i} p_{k-1}\right)\right. \text {; } \\
& y_{k}=y_{k-1}+\alpha_{k-1} p_{k-1} ; \\
& r_{k}=r_{k-1}-\alpha_{k-1} L_{i} p_{k-1} \text {; } \\
& \sigma_{k}=\left(r_{k}, r_{k}\right)_{i} \text {; } \\
& \text { if } \sigma_{k}=0 \text { then }\left\{y_{m_{i}}=y_{k} \text {; goto } 2.3\right\} \text {; } \\
& \beta_{k}=\sigma_{k} / \sigma_{k-1} \text {; } \\
& p_{k}=r_{k}+\beta_{k} p_{k-1} \\
& \text { \} the end of the iteration; }
\end{aligned}
$$

2.3. Set $v_{i}=y_{m_{i}}$;

\} the end of the level $i$;

\} the end of the algorithm.

We shall study the convergence properties of the CCG-algorithm under the assumption that the operators $L_{i}$, for $i=0,1, \cdots, l$, are self-adjoint and positive definite, i.e., for $i=0,1, \cdots, l$ we have

$$
\left(L_{i} u, v\right)_{i}=\left(u, L_{i} v\right)_{i}, \quad\left(L_{i} u, u\right)_{i} \geq \alpha_{i}(u, u)_{i}, \quad \alpha_{i}>0, \quad \forall u, v \in M_{i} .
$$

Moreover, we assume that the operator $L_{i-1}: M_{i-1} \rightarrow M_{i-1}$ on the lower level can be represented by means of the operator $L_{i}: M_{i} \rightarrow M_{i}$ and the transfer operators $I_{i-1}: M_{i-1} \rightarrow M_{i}, I_{i-1}^{*}: M_{i} \rightarrow M_{i-1}$ in the form

$$
L_{i-1}=I_{i-1}^{*} L_{i} I_{i-1} .
$$

Note that the adjoint operator $I_{i-1}^{*}: M_{i} \rightarrow M_{i-1}$, for $i=1,2, \cdots, l$, is defined by

$$
\left(I_{i-1}^{*} v, w\right)_{i-1}=\left(v, I_{i-1} w\right)_{i} \quad \forall v \in M_{i}, w \in M_{i-1} .
$$

We introduce a scale of norms on $M_{i}$ by

$$
\|\mid u\|_{i}^{(\alpha)}:=\sqrt{\left(L_{i}^{\alpha} u, u\right)_{i}}, \quad u \in M_{i},
$$

with $\alpha \in(-\infty, \infty)$. In order to simplify the notation, we write

$$
\|u\|\left\|_{i}:=\right\| u\left\|_{i}^{(1)}, \quad\right\| u\left\|_{i}:=\right\|\|u\|_{i}^{(0)}, \quad u \in M_{i} .
$$

The operator norm induced by $\left|\|\cdot \mid\| \|_{i}\right.$ for an operator $B: M_{i} \rightarrow M_{i}$ is given by

$$
\|B\|_{i}=\sup _{u \in M_{i} \backslash\{0\}} \frac{\|B u \mid\|_{i}}{\|\| u \mid \|_{i}} .
$$


On a fixed level $i \in\{1, \cdots, l\}$ we apply the CG-algorithm to reduce the error $u_{i}-w_{i}$ of the initial guess $w_{i}$ for the exact solution $u_{i}$ of problem (2.4). After $m_{i}$ steps we get the error

$$
u_{i}-v_{i}:=B_{i}\left(u_{i}-w_{i}\right)
$$

of the final approximation $v_{i}$ on level $i$. In this way we define the operator $B_{i}$ : $M_{i} \rightarrow M_{i}$ of error reduction on level $i$. This operator can be represented as a polynomial in $L_{i}$ :

$$
B_{i}=P_{i}\left(L_{i}\right)=I+\sum_{k=1}^{m_{i}} a_{k} L_{i}^{k}
$$

with coefficients which depend on the parameters $\sigma_{0}, \cdots, \sigma_{m_{i}}, \alpha_{0}, \cdots, \alpha_{m_{i-1}}$ given in the definition of the CCG algorithm (see [10]). Here and in the following we denote by $I$ the identity in the corresponding space. From $[10$ we recall the wellknown optimality property of the CG-algorithm:

Lemma 2.1. Among all polynomials of the form (2.8) with arbitrary coefficients $a_{k}$ the conjugate-gradient method minimizes the error $u_{i}-v_{i}$ of the final approximation $v_{i}$ in the norm $\left|\|\cdot \mid\|_{i}\right.$ for a fixed given initial guess $w_{i}$.

\section{Optimal POLYNOMials}

For estimating the norm of the error-reduction operator $B_{i}$ on the level $i$, we consider polynomials $q_{m}$ of degree $m$ with $q_{m}(0)=1$. These polynomials can be written in the form

$$
q_{m}(x)=\prod_{k=1}^{m}\left(1-\mu_{k} x\right),
$$

with parameters $\mu_{k} \neq 0$ for $k=1, \ldots, m$. Note that the polynomial $P_{i}$ that defines the error-reduction operator $B_{i}$ on the level $i$ has the same structure. We shall show that on a given compact set $[0, d]$ the parameters $\mu_{k}$, for $k=1, \ldots, m$, can be chosen in such a way that the resulting polynomial satisfies certain optimality properties.

Lemma 3.1. For any $\gamma>0$ and any $d>0$, there exist parameters $\mu_{k}$, for $k=$ $1, \ldots, m$, such that the polynomial defined by (3.1) satisfies

$$
\max _{0 \leq x \leq d}\left|q_{m}(x)\right| \leq 1
$$

and

$$
\max _{0 \leq x \leq d}\left|x^{\gamma / 2} q_{m}(x)\right| \leq \eta_{\gamma}(m) d^{\gamma / 2},
$$

where $\eta_{\gamma}(m)$ is independent of $d$ and tends to 0 if $m$ tends to infinity.

Proof. We consider the minimization problem

Find parameters $\mu_{1}, \cdots, \mu_{m}$ such that

$$
M\left(\mu_{1}, \cdots, \mu_{m}\right):=\max _{0 \leq x \leq d}\left|\sqrt{x} q_{m}(x)\right|
$$

becomes minimal. 
In [13, §4.1], it has been proved that the solution of this problem defines the polynomial $\bar{q}_{m}$ with

$$
\sqrt{x} \bar{q}_{m}(x)=(-1)^{m} p_{m} \cos ((2 m+1) \arccos \sqrt{x / d}), \quad p_{m}=\sqrt{d} /(2 m+1) .
$$

One can check that $\bar{q}_{m}$ is a polynomial in $x$ of degree $m$ with zeros at $\bar{x}_{k}$ and whose parameters $\mu_{k}$ are given by

$$
\bar{\mu}_{k}=\frac{1}{\bar{x}_{k}}=\frac{1}{d} \cos ^{-2} \frac{\pi(2 k+1)}{2(2 m+1)}, \quad \text { for } k=0, \cdots, m-1 .
$$

In [13, §4.1] it has also been proved that

$$
\left|\bar{q}_{m}(x)\right| \leq 1 \quad \forall x \in[0, d] .
$$

It follows directly from 3.5 that

$$
\left|\sqrt{x} \bar{q}_{m}(x)\right| \leq p_{m} \quad \forall x \in[0, d] .
$$

Now let us first consider the case $\gamma \in(0,1]$. Using (3.7) and (3.8), we get

$$
\left|x^{\gamma / 2} \bar{q}_{m}(x)\right| \leq\left|\sqrt{x} \bar{q}_{m}(x)\right|^{\gamma} \leq d^{\gamma / 2} /(2 m+1)^{\gamma} \quad \forall x \in[0, d] .
$$

Thus we have shown that there are parameters $\mu_{k}$ in (3.1) such that the estimates (3.2) and (3.3) hold with $\eta_{\gamma}(m)$ defined by

$$
\eta_{\gamma}(m)=\frac{1}{(2 m+1)^{\gamma}}, \quad \text { for } \gamma \in(0,1] .
$$

Next we consider the case $\gamma>1$ and put

$$
r=-[-\gamma]=\left\{\begin{array}{l}
\gamma \text { if } \gamma \text { is an integer } \\
{[\gamma]+1 \text { otherwise. }}
\end{array}\right.
$$

Any integer $m$ can be decomposed in the form

$$
m=t r+s,
$$

where $t=[m / r]$ and $0 \leq s \leq r-1$. Then the polynomial

$$
\overline{\bar{q}}_{m}(x)=\bar{q}_{t}^{r-s}(x) \bar{q}_{t+1}^{s}(x)
$$

is of the form (3.1), with $t$ parameters

$$
\overline{\bar{\mu}}_{i}=\frac{1}{d} \cos ^{-2} \frac{\pi(2 i+1)}{2(2 t+1)}, \quad \text { for } i=0, \cdots, t-1,
$$

of multiplicity $r-s$ and $t+1$ parameters

$$
\overline{\bar{\mu}}_{j}=\frac{1}{d} \cos ^{-2} \frac{\pi(2 j+1)}{2(2 t+3)}, \quad \text { for } j=0, \cdots, t,
$$

of multiplicity $s$. Applying (3.7) to $\bar{q}_{t}$ and $\bar{q}_{t+1}$, we get

$$
\left|\overline{\bar{q}}_{m}(x)\right|=\left|\bar{q}_{t}(x)\right|^{r-s}\left|\bar{q}_{t+1}\right|^{s} \leq 1 \quad \forall x \in[0, d] .
$$

In order to show (3.3) we use (3.8) for $\bar{q}_{t}$ and $\bar{q}_{t+1}$, and obtain

$$
\left|x^{\gamma / 2 r} \bar{q}_{t}(x)\right| \leq\left|\sqrt{x} \bar{q}_{t}(x)\right|^{\gamma / r} \leq\left(\frac{\sqrt{d}}{2 t+1}\right)^{\gamma / r} \quad \forall x \in[0, d]
$$

and

$$
\left|x^{\gamma / 2 r} \bar{q}_{t+1}(x)\right| \leq\left|\sqrt{x} \bar{q}_{t+1}(x)\right|^{\gamma / r} \leq\left(\frac{\sqrt{d}}{2 t+3}\right)^{\gamma / r} \quad \forall x \in[0, d] .
$$


Consequently,

$$
\left|x^{\gamma / 2} \overline{\bar{q}}_{m}(x)\right| \leq\left|x^{\gamma / 2 r} \bar{q}_{t}(x)\right|^{r-s}\left|x^{\gamma / 2 r} \bar{q}_{t+1}(x)\right|^{s} \leq d^{\gamma / 2} \eta_{\gamma}(m) \quad \forall x \in[0, d],
$$

where

$$
\eta_{\gamma}(m)=\frac{1}{(2 t+1)^{\gamma(r-s) / r}(2 t+3)^{\gamma s / r}} \quad \forall x \in[0, d] .
$$

Note that the function $\eta_{\gamma}(\cdot)$ is monotonically decreasing with respect to $m=t r+s$. Moreover, if $s=0$ (i.e., if $m$ is a multiple of $r$ ) we have

$$
\eta_{\gamma}(m)=\frac{1}{(2 m / r+1)^{\gamma}}=O\left(m^{-\gamma}\right) \text {. }
$$

Therefore $\eta_{\gamma}(m)$ tends to 0 when $m \rightarrow \infty$. Thus, setting $\mu_{k}$ in (3.1) equal to the values in (3.11) and (3.12) with their corresponding multiplicities, we get (3.3) for the case $\gamma>1$ also.

We set $d=\lambda_{i}^{*}$, where $\lambda_{i}^{*}$ denotes the largest eigenvalue of the operator $L_{i}$ in the space $M_{i}$, and choose the parameters $\mu_{k}$ as in the proof of Lemma 3.1. Then the optimal polynomial $q_{m}$ defines the auxiliary operator $S_{i, m}$ by

$$
S_{i, m}=q_{m}\left(L_{i}\right)=\prod_{k=1}^{m}\left(I-\mu_{k} L_{i}\right)
$$

which majorizes the error-reduction operator $B_{i}$ on level $i$ owing to Lemma 2.1.

Lemma 3.2. Let the operator $L_{i}$ be self-adjoint and positive definite. Then for any $\gamma>0$, we have the inequalities

$$
\left\||| S_{i, m} w\left|\left\|_{i} \leq\left(\lambda_{i}^{*}\right)^{\gamma / 2} \eta_{\gamma}(m)\right\|\right||w|\right\|_{i}^{(1-\gamma)} \quad \forall w \in M_{i}
$$

and

$$
\left\||| S_{i, m} w\right\|_{i} \leq\left|\|w \mid\|_{i} \quad \forall w \in M_{i},\right.
$$

where the function $\eta_{\gamma}$ is independent of $d$ and tends to 0 if $m$ tends to infinity.

Proof. We can assume that the set of eigenvectors $\left\{\varphi_{j}\right\}_{j=1}^{n_{i}}$ of the eigenvalue problem

$$
L_{i} \varphi_{j}=\lambda_{j} \varphi_{j}, \quad \text { for } j=1, \cdots, n_{i}, \quad \text { where } n_{i}=\operatorname{dim} M_{i},
$$

is orthonormal with respect to the inner product $(\cdot, \cdot)_{i}$, i.e.,

$$
\left(\varphi_{j}, \varphi_{k}\right)_{i}=\delta_{j k}, \quad \text { for } j, k=1, \ldots, n_{i},
$$

where $\delta_{j k}$ is Kronecker's symbol. Then, using the basis representation

$$
w=\sum_{j=1}^{n_{i}} \alpha_{j} \varphi_{j}
$$

of $w \in M_{i}$, we get

$$
\left(\left|\|w \mid\|_{i}^{(1-\gamma)}\right)^{2}=\sum_{j=1}^{n_{i}} \lambda_{j}^{1-\gamma} \alpha_{j}^{2}\right.
$$

and

$$
\left|\left\|S_{i, m} w \mid\right\|_{i}^{2}=\sum_{j=1}^{n_{i}} \lambda_{j} q_{m}^{2}\left(\lambda_{j}\right) \alpha_{j}^{2}\right.
$$


From (3.3) we obtain

$$
\begin{aligned}
\sum_{j=1}^{n_{i}} \lambda_{j} q_{m}^{2}\left(\lambda_{j}\right) \alpha_{j}^{2} & \leq \eta_{\gamma}^{2}(m)\left(\lambda_{i}^{*}\right)^{\gamma} \sum_{j=1}^{n_{i}} \lambda_{j}^{1-\gamma} \alpha_{j}^{2} \\
& =\eta_{\gamma}^{2}(m)\left(\lambda_{i}^{*}\right)^{\gamma}\left(\||| w \mid\|_{i}^{(1-\gamma)}\right)^{2},
\end{aligned}
$$

which implies (3.17). Using (3.2), we get immediately

$$
\sum_{j=1}^{n_{i}} \lambda_{j} q_{m}^{2}\left(\lambda_{j}\right) \alpha_{j}^{2} \leq \sum_{j=1}^{n_{i}} \lambda_{j} \alpha_{j}^{2}=\|\| w \|_{i}^{2},
$$

which implies (3.18).

\section{The Algebraic CONVERGENCE THEOREM}

In order to formulate our abstract convergence result, we assume that the following criterion is satisfied:

There exist constants $c^{*}>0$ and $\gamma>0$ such that for $i=1, \cdots, l$ we have the following relation between two neighbouring solutions $u_{i-1}$ and $u_{i}$ of the problems (2.4):

$$
\left\|\left|u_{i}-I_{i-1} u_{i-1}\right|\right\|_{i}^{(1-\gamma)} \leq c^{*}\left(\lambda_{i}^{*}\right)^{-\gamma / 2} \mid\left\|u_{i}-I_{i-1} u_{i-1}\right\|_{i} .
$$

Note that this inequality can be proved not only in the case of $H^{1+\lambda}$-regularity with $\lambda \in(0,1]$, but also for $\lambda>1$ when for example second-order finite elements are used.

Theorem 4.1. Let the operators $L_{i}$, for $i=0, \ldots, l$, be self-adjoint, positive definite and satisfy

$$
L_{i-1}=I_{i-1}^{*} L_{i} I_{i-1} .
$$

We assume that the convergence criterion (4.1) holds for some $\gamma>0$. Then, for each level $i$, where $i=1, \ldots, l$, the approximate solution $v_{i}$ of the CCG-algorithm satisfies the inequality

$$
\left\|\left|u_{i}-v_{i}\right|\right\|_{i} \leq c^{*} \sum_{j=1}^{i} \eta_{\gamma}\left(m_{j}\right)||\left|u_{j}-I_{j-1} u_{j-1}\right| \|_{j},
$$

where the constant $c^{*}$ and the function $\eta_{\gamma}$ are independent of $i$ and $u_{j}$ for $j=$ $1, \ldots, i$.

Proof. Let us denote the iteration error of the CCG-algorithm at level $i$ after $m_{i}$ steps by

$$
\varepsilon_{i}=u_{i}-v_{i} \quad \forall i=0,1, \cdots, l .
$$

Using the definition of the error-reduction operator $B_{i}$, we have

$$
\varepsilon_{i}=B_{i}\left(u_{i}-w_{i}\right),
$$


where $w_{i}$ is the initial guess for the CG-algorithm on level $i$. The polynomial $q_{m}(\cdot)$ that defines $S_{i, m_{i}}(\cdot)$ has the form (2.8) with some coefficients altered. The minimization property of the CG-algorithm (Lemma 2.1) implies that

$$
\begin{aligned}
\left\|\varepsilon_{i} \mid\right\|_{i} & =\left\|\mid B_{i}\left(u_{i}-w_{i}\right)\right\| \|_{i} \\
& \leq\left\|\mid S_{i, m_{i}}\left(u_{i}-w_{i}\right)\right\|_{i} \\
& \leq\left\||| S_{i, m_{i}}\left(u_{i}-I_{i-1} u_{i-1}\right)\right\|\left\|_{i}+\right\| \mid S_{i, m_{i}} I_{i-1} \varepsilon_{i-1}\|\|_{i} .
\end{aligned}
$$

Taking into consideration (3.17) and (4.1), we can estimate the first term in the right-hand side of (4.3):

$$
\begin{aligned}
\left|\left\|S_{i, m_{i}}\left(u_{i}-I_{i-1} u_{i-1}\right) \mid\right\|_{i}\right. & \leq\left(\lambda_{i}^{*}\right)^{\gamma / 2} \eta_{\gamma}\left(m_{i}\right)\left|\left\|u_{i}-I_{i-1} u_{i-1} \mid\right\|_{i}^{(1-\gamma)}\right. \\
& \leq c^{*} \eta_{\gamma}\left(m_{i}\right)\left\|u_{i}-I_{i-1} u_{i-1}\right\| \|_{i} .
\end{aligned}
$$

To estimate the second term, we use (3.18):

$$
\left.\left\|\left|S_{i, m_{i}} I_{i-1} \varepsilon_{i-1}\right|\right\|\right|_{i} \leq\left\||| I_{i-1} \varepsilon_{i-1} \mid\right\|_{i},
$$

and by (2.6) we have

$$
\begin{aligned}
\left.\left\|I_{i-1} \varepsilon_{i-1}\right\|\right|_{i} ^{2} & =\left(I_{i-1}^{*} L_{i} I_{i-1} \varepsilon_{i-1}, \varepsilon_{i-1}\right)_{i-1} \\
& =\left(L_{i-1} \varepsilon_{i-1}, \varepsilon_{i-1}\right)_{i-1} \\
& =\left.\left\|\varepsilon_{i-1}\right\|\right|_{i-1} ^{2} .
\end{aligned}
$$

Thus we obtain

$$
\left|\left\|\varepsilon _ { i } \left|\left\|_{i} \leq c^{*} \eta_{\gamma}\left(m_{i}\right)\left|\left\|u_{i}-\left.I_{i-1} u_{i-1}||\right|_{i}+\left|\left\|\varepsilon_{i-1} \mid\right\|_{i-1},\right.\right.\right.\right.\right.\right.\right.
$$

from which the statement of the theorem follows by induction on $i$.

Remark 4.2. Each term in the sum (4.2),

$$
\frac{c^{*}}{2 m_{j}+1} \mid\left\|u_{j}-I_{j-1} u_{j-1}\right\| \|_{j},
$$

can be considered as the error contribution at the corresponding level $j$ of the CCG-algorithm. Since $c^{*}$ is independent of $j$ and $m_{j}$, we can reduce this error contribution by taking a suffiently large number of smoothing steps $m_{j}$. Later we shall see that asymptotically the size of the term ||$\left|u_{j}-I_{j-1} u_{j-1}\right| \|_{j}$ also decreases as the level $j$ increases. This is important, since the complexity of the CG-iteration increases with $j$.

\section{The Boundary VAlue PROBlem}

Let us consider the following Dirichlet problem in an open bounded polygon $\Omega \subset \mathbf{R}^{2}$ with boundary $\Gamma=\partial \Omega$ :

$$
\begin{gathered}
-\sum_{i, j=1}^{2} \partial_{i}\left(a_{i j} \partial_{j} u\right)+b u=f \text { in } \Omega, \\
u=0 \text { on } \Gamma,
\end{gathered}
$$

where the coefficients and the right-hand side of (5.1) satisfy the conditions

$$
\left\{\begin{array}{l}
\partial_{k} a_{i j} \in L_{q}(\Omega), \quad q>2, \quad i, j, k=1,2 ; \quad a_{12}=a_{21} \quad \text { on } \bar{\Omega} \\
b \in L_{2}(\Omega) ; \quad b \geq 0 \quad \text { on } \bar{\Omega} ; \quad \text { there are } \nu_{2} \geq \nu_{1}>0 \text { such that } \\
\nu_{1} \sum_{i=1}^{2} \xi_{i}^{2} \leq \sum_{i, j=1}^{2} a_{i j}(\cdot) \xi_{i} \xi_{j} \leq \nu_{2} \sum_{i=1}^{2} \xi_{i}^{2} \quad \forall \xi_{i} \in \mathbf{R} \quad \text { on } \bar{\Omega} .
\end{array}\right.
$$


We shall use the standard notation of Sobolev spaces $H^{s}(\Omega)$ equipped with the norm $\|\cdot\|_{s, \Omega}$ for any integer $s \geq 0$. The space $H^{0}(\Omega)$ coincides with the space $L_{2}(\Omega)$, and $H_{0}^{1}(\Omega)$ is the subspace of $H^{1}(\Omega)$ that is the closure of the set $C_{0}^{\infty}(\Omega)$ of infinite differentiable functions with compact support in $\Omega$. With this notation the problem (5.1) - (5.2) can be formulated in its weak form:

Find $u \in H_{0}^{1}(\Omega)$ such that

$$
a(u, v)=(f, v)_{\Omega} \quad \forall v \in H_{0}^{1}(\Omega),
$$

where the bilinear form $a(\cdot, \cdot)$ and the linear form $(f, \cdot)$ are given by

$$
a(u, v)=\int_{\Omega}\left(\sum_{i, j=1}^{2} a_{i j} \partial_{j} u \partial_{i} v+b u v\right) d x, \quad(f, v)_{\Omega}=\int_{\Omega} f v d x .
$$

It is known that under the assumptions (5.3) the problem (5.4), (5.5) has a unique solution [8]. Under the assumptions that $f$ belongs to $L_{2}(\Omega)$ and that $\Omega$ is convex the solution is $H^{2}$-regular. This regular case has been studied in detail in [12]. Here we are interested in the more general case of a non-convex polygon. To simplify the presentation we consider only the case of one re-entrant corner with inner angle $\theta>\pi$ at the origin $(0,0)$.

In order to describe the type of regularity loss, let us take a positive $r_{0}$ in such a way that the circumference of the circle with center $(0,0)$ and radius $r_{0}$ cuts only a sector $\omega$ from the domain $\Omega$. Then we introduce polar coordinates $(r, \varphi)$, where $x_{1}=r \cos \varphi$ and $x_{2}=r \sin \varphi$, such that sector $\omega$ is described by $0<r<r_{0}$ and $0<\varphi<\theta$. The singular behaviour of the solution in these coordinates is characterized by the following function:

$$
\tilde{w}(r, \varphi)=r^{\mu} \tilde{\xi}(r) \sin \pi \varphi / \theta,
$$

where the constant $\mu \in(1 / 2,1)$ can be given in explicit form [9, 6] for example in the case of Poisson's equation we have $\mu=\pi / \theta$. The cutoff function $\tilde{\xi}(r) \in$ $C^{\infty}[0, \infty)$ is given by

$$
\tilde{\xi}(r)=\left\{\begin{array}{l}
1 \quad \text { if } \quad r \in\left[0, r_{0} / 2\right], \\
\text { monotone if } r \in\left[r_{0} / 2, r_{0}\right], \\
0 \quad \text { if } \quad r \in\left[r_{0}, \infty\right) .
\end{array}\right.
$$

Using the singular function (5.6), we can represent the solution of (5.1)-(5.2) in the form

$$
u(x)=v(x)+\sigma w(x),
$$

where $w\left(x_{1}, x_{2}\right)=w(r \cos \varphi, r \sin \varphi)=\tilde{w}(r, \varphi)$, and $\sigma$ and $v$ denote a constant and the regular part of the solution, respectively, that satisfy

$$
|\sigma|+\|v\|_{2, \Omega} \leq c\|f\|_{0, \Omega} .
$$

The regularity properties of the solution $u$ can also be described by special spaces with weighted norms [1]. For this purpose, we introduce the weighting function

$$
\Phi_{\beta}\left(x_{1}, x_{2}\right)=\Phi_{\beta}(r \cos \varphi, r \sin \varphi)=\tilde{\Phi}_{\beta}(r, \varphi)=r^{\beta} \quad \text { for } \beta \in(-\infty, \infty)
$$


and denote by $H^{m, \beta}(\Omega)$, for $m \geq 0$ and $0 \leq \beta<1$, the closure of $C^{\infty}(\Omega)$ in the norm $\|\cdot\|_{m, \beta}$ defined by

$$
\begin{aligned}
\|u\|_{m, \beta}^{2} & =\|u\|_{m-1}^{2}+\int_{\Omega} \Phi_{\beta}^{2} \sum_{m_{1}+m_{2}=m}\left(\partial_{1}^{m_{1}} \partial_{2}^{m_{2}} u\right)^{2} d x, \\
m & \geq 1, \quad m_{1} \geq 0, \quad m_{2} \geq 0 \\
\|u\|_{0, \beta}^{2} & =\int_{\Omega} \Phi_{\beta}^{2} u^{2} d x .
\end{aligned}
$$

Then we have the following regularity result [7, Theorem 1.1]:

Theorem 5.1. Suppose that $1-\mu<\beta<1$ and

$$
f \in H^{0, \beta}(\Omega) .
$$

Then the solution $u$ of (5.4) belongs to $H^{2, \beta}(\Omega)$, and there is a constant $c_{1}>0$, which is independent of $f$, such that

$$
\|u\|_{2, \beta} \leq c_{1}\|f\|_{0, \beta} .
$$

Remark 5.2. Note that $L_{2}(\Omega) \subset H^{0, \beta}(\Omega)$ for $\beta \geq 0$. Therefore the condition

$$
f \in L_{2}(\Omega)
$$

is sufficient for (5.11), and a positive constant $c_{2}$ exists such that

$$
\|f\|_{0, \beta} \leq c_{2}\|f\|_{0} .
$$

\section{THE MESh REFinEMENT STRATEGY}

Standard finite element triangulations result in optimal convergence rates, provided that the solution is sufficiently regular. A reduction of the convergence rate can be observed both theoretically and numerically when the solution is not $\mathrm{H}^{2}$ regular. Consequently special mesh refinement strategies have been developed to guarantee optimal convergence rates [9, 1, 14. Standard techniques for adapting the grid in the neighbourhood of singular points lead to a family of meshes with optimal order of convergence, but not necessarily to a family of nested finite element spaces (see, e.g., [9]). In this section we derive a special mesh refinement technique which guarantees both optimal order of convergence and a nested family of finite element spaces.

We shall follow a technique described in 13 . Let us start with an initial admissible triangulation $\mathcal{F}_{0}$ of $\Omega$ into closed triangles, i.e., each pair of triangles has either no common points or a common vertex or a common edge. In order to define the refinement near the singular point $A=(0,0)$, we introduce the refinement index $\rho \geq 1$. Then every initial triangle is divided into 4 finer ones. If a triangle of refinement level $i$, where $i=0,1, \cdots, l-1$, does not contain the origin $(0,0)$, it is subdivided into 4 triangles by connecting the midpoints of its edges. This type of refinement is called a regular partition of the domain. Now let $\triangle A B_{i} C_{i}$ be a triangle of refinement level $i$ and have a vertex $A=(0,0)$ (see Figure 1). We denote by $b_{0}$ the length of the perpendicular from $A$ to the opposite side $B_{0} C_{0}$ of the initial triangle $\triangle A B_{0} C_{0}$. Then we construct the straight segment $B_{i+1} C_{i+1}$ parallel to $B_{0} C_{0}$ at a distance $b_{0} 2^{-(i+1) \rho}$ from $A$, with ends $B_{i+1}, C_{i+1}$ on the edges $A B_{0}$, $A C_{0}$ respectively. The midpoint of $B_{i} C_{i}$ is denoted by $A_{i+1}$. Connecting the points 


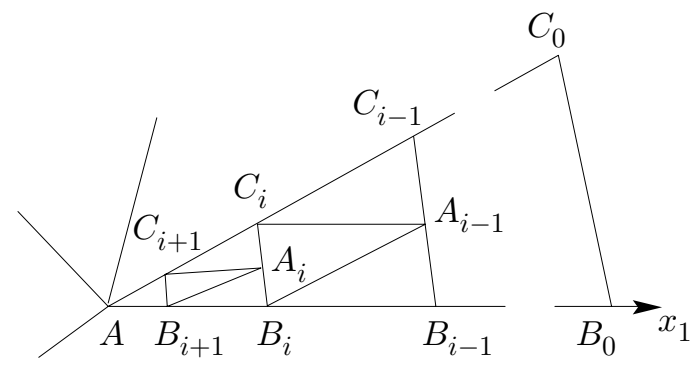

FIGURE 1. Irregular partition of triangle.

$A_{i+1}, B_{i+1}, C_{i+1}$, we get 4 finer triangles of the triangulation $\mathcal{F}_{i+1}$ at refinement level $(i+1)$. This type of refinement is called an irregular partition.

Thus, starting with the initial admissible triangulation $\mathcal{F}_{0}$ of $\Omega$, we end up with a family of admissible triangulations $\mathcal{F}_{i}$ for any $i$, where $i=1,2, \cdots$. Before we show that $\mathcal{F}$ is a triangulation of the type considered in [1], we note an important property of the locally refined meshes constructed above. If we define the maximal length of all edges of the triangulation $\mathcal{F}_{i}$ by $h_{i}$, then the relation

$$
h_{i}=h_{1} 2^{-i+1}
$$

holds true for $i=1,2, \cdots$. This can be seen in the following way. On the triangulation $\mathcal{F}_{i}$, for $i=0,1, \cdots$, all edges of triangles sharing $A$ as a vertex have length less than or equal to $h_{i}$. Now let us consider the next triangulation $\mathcal{F}_{i+1}$. Since the distance from each parallel line $B_{i} C_{i}$ to $A$ is $b_{0} 2^{-(i+1) \rho}$, for $i=0,1, \cdots$, we can show by an elementary argument that

$$
\begin{aligned}
\left|C_{i+1} C_{i}\right| & \leq\left|C_{i} C_{i-1}\right| / 2, \quad\left|C_{i+1} A_{i}\right| \leq\left|C_{i} A_{i-1}\right| / 2 . \\
\left|B_{i+1} A_{i}\right| & \leq\left|B_{i} A_{i-1}\right| / 2, \quad\left|B_{i+1} B_{i}\right| \leq\left|B_{i} B_{i-1}\right| / 2 .
\end{aligned}
$$

Therefore we have

$$
h_{i+1}=h_{i} / 2 \text {. }
$$

Thus by induction we get (6.1). To classify the properties of the triangulation we shall use the definition given in [1]:

A triangulation $\mathcal{F}$ is said to be of type $(h, \gamma, L)$ if it satisfies the following three properties:

(i): for any triangle $\triangle \in \mathcal{F}$ and for any angle $\alpha$ of $\triangle$, we have

$$
\alpha \geq L^{-1}
$$

(ii): if $\Phi_{\gamma} \neq 0$ on $\triangle$, then

$$
L^{-1} \Phi_{\gamma}(x) \leq d_{\triangle} / h \leq L \Phi_{\gamma}(x),
$$

where $d_{\triangle}=\sup \{|x-y|: x, y \in \triangle\}$ is the diameter of $\triangle$;

(iii): if $\Phi_{\gamma}=0$ at some point of $\triangle$, then

$$
L^{-1} \sup _{x \in \triangle} \Phi_{\gamma}(x) \leq d_{\triangle} / h \leq L \sup _{x \in \triangle} \Phi_{\gamma}(x) .
$$

Lemma 6.1. Let $\rho$ be the refinement index and $\gamma=(\rho-1) / \rho$. Then each triangulation $\mathcal{F}_{i}$ is of type $\left(h_{i}, \gamma, L\right)$ with a constant $L$ independent of $i$. 
Proof. First, we note that in each initial triangle there are only 4 different types of geometrically similar triangles at any level of triangulation. They are similar to the triangles of $\mathcal{F}_{1}$. Therefore their angles are independent of the level number $i$. Thus (6.2) holds true with

$$
L \geq L_{0}=\max _{\triangle \in \mathcal{F}_{1}} \alpha^{-1} .
$$

Now let $x \in \triangle_{j} \in \mathcal{F}_{j}$ and let $\Phi_{\gamma} \neq 0$ on $\triangle_{j}$, i.e., the point $A$ does not belong to the $\triangle_{j}$. We consider all triangles $\triangle_{k}$ on lower levels that contain this $x$ :

$$
x \in \triangle_{j} \subset \cdots \subset \triangle_{i+1} \subset \triangle_{i} \subset \cdots \subset \triangle_{0} \in \mathcal{F}_{0} .
$$

There are two cases. First, $\triangle_{0}$ does not contain $A$. This means that $\Phi_{\gamma} \geq c$ on $\triangle_{0}$ (and in particular on $\triangle_{j}$ ) for some constant $c$ that is independent of $j$. Moreover, we have $d_{\triangle_{j}}=d_{\triangle_{1}} h_{j} / h_{1}$ for $j=1,2, \cdots$. Both these facts follow from (6.3) for this type of triangle. Second, $\triangle_{0}$ contains $A$. Then there is an index $i$ such that

$$
A \notin \triangle_{i+1} \text { but } A \in \triangle_{i} .
$$

The notation used in the following is given in Figure 1, The statement (6.7) implies that $x$ belongs to the trapezium $C_{i+1} B_{i+1} B_{i} C_{i}$. Therefore the inequality $|x-A| \geq$ $b_{0} 2^{-(i+1) \rho}$ holds true. Hence, we have

$$
b_{0}^{\gamma} 2^{-(i+1)(\rho-1)} \leq \Phi_{\gamma}(x) \leq d_{\triangle_{i}}^{\gamma}
$$

by definition of $\Phi_{\gamma}$. On the one hand, $d_{\triangle_{i+1}}$ can be estimated from above by

$$
d_{\triangle_{i+1}} \leq d_{\triangle_{i}}=d_{\triangle_{0}} 2^{-i \rho}
$$

Taking

$$
L \geq L_{1}=d_{\triangle_{0}} h_{1}^{-1} b_{0}^{-\gamma} 2^{\rho-1}
$$

we get

$$
d_{\triangle_{i+1}} / h_{i+1} \leq L_{1} \Phi_{\gamma}(x) \leq L \Phi_{\gamma}(x) .
$$

On the other hand, $d_{\triangle_{i+1}}$ can be estimated from below by

$$
d_{\triangle_{i+1}} \geq d_{\triangle_{i}} / 2^{\rho}=2^{-(i+1) \rho} d_{\triangle_{0}} .
$$

Taking

$$
L^{-1} \leq L_{2}^{-1}=d_{\triangle_{0}}^{1-\gamma} h_{1}^{-1} 2^{-\rho}
$$

we get the inequalities

$$
d_{\triangle_{i+1}} / h_{i+1} \geq L_{2}^{-1} \Phi_{\gamma}(x) \geq L^{-1} \Phi_{\gamma}(x) .
$$

Thus (6.3) is proved at the level $i+1$. At the higher levels $j>i+1$, we have

$$
d_{\triangle_{j}} / h_{j}=d_{\triangle_{i+1}} / h_{i+1}
$$

by construction. Therefore (6.10) and (6.11) give us

$$
L^{-1} \Phi_{\gamma}(x) \leq L_{2}^{-1} \Phi_{\gamma}(x) \leq d_{\triangle_{j}} / h_{j} \leq L_{1} \Phi_{\gamma}(x) \leq L \Phi_{\gamma}(x)
$$

for all $j$ when $\Phi_{\gamma} \neq 0$ on $\triangle_{j}$.

Finally, let $\Phi_{\gamma}(x)=0$ at some point $x \in \triangle$. This means that $x=A$. For instance, let $x=A \in \triangle_{i+1}=\triangle A B_{i+1} C_{i+1}$ in Figure1 By analogy with (6.8), we have

$$
b_{0}^{\gamma} 2^{-(i+1)(\rho-1)} \leq \sup _{\triangle_{i+1}} \Phi_{\gamma} \leq d_{\triangle_{i+1}}^{\gamma}
$$


Let us choose

$$
L \geq L_{3}=d_{\triangle_{0}} /\left(2 h_{1} b_{0}^{\gamma}\right)
$$

Since

$$
d_{\triangle_{i+1}}=d_{\triangle_{0}} 2^{-(i+1) \rho},
$$

we get

$$
d_{\triangle_{i+1}} / h_{i+1} \leq L_{3} \sup _{\triangle_{i+1}} \Phi_{\gamma} \leq L \sup _{\triangle_{i+1}} \Phi_{\gamma} .
$$

Then, for $L$ satisfying

$$
L^{-1} \leq L_{4}^{-1}=d_{\triangle_{0}}^{1-\gamma} / 2 h_{1},
$$

and taking into consideration (6.13) and (6.14), we have

$$
d_{\triangle_{i+1}} / h_{i+1} \geq L_{4}^{-1} \sup _{\triangle_{i+1}} \Phi_{\gamma} \geq L \sup _{\triangle_{i+1}} \Phi_{\gamma} .
$$

Therefore, (6.2)-(6.4) are proved if $L$ is sufficiently large; more precisely, the choice

$$
L=\max \left\{L_{0}, \cdots, L_{4}\right\}
$$

is sufficient to guarantee (6.2)-(6.4).

Thus conditions (i)-(iii) of [1] are satisfied and we can use its results. For example, there is a constant $c_{3}$ independent of $h_{i}$ such that the number $n_{i}$ of interior vertices in $\mathcal{F}_{i}$ can be estimated in the following way:

$$
n_{i} \leq c_{3} h_{i}^{-2} .
$$

Now we derive the Galerkin approximation based on the triangulation described above. We denote by $\bar{\Omega}_{i}$ the set of all nodes of the triangulation $\mathcal{F}_{i}$, and by $\Omega_{i}$ the set of all interior nodes. For each node $y \in \Omega_{i}$, we define the basis function $\varphi_{y}^{i} \in H_{0}^{1}(\Omega)$ by requiring it to be linear on each triangle of the triangulation $\mathcal{F}_{i}$, to equal 1 at the node $y$ and to equal 0 at every other node $z \in \bar{\Omega}_{i}$. We denote the linear span of these functions by

$$
\mathcal{H}^{i}=\operatorname{span}\left\{\varphi_{y}^{i}: y \in \Omega_{i}\right\} .
$$

Considering (5.4) on the subspace $\mathcal{H}^{i} \subset H_{0}^{1}(\Omega)$, we get the discrete problem:

Find $\tilde{u}_{i} \in \mathcal{H}^{i}$ such that

$$
a\left(\tilde{u}_{i}, v\right)=(f, v)_{\Omega} \quad \forall v \in \mathcal{H}^{i} .
$$

Let $M_{i}$ be the $n_{i}$-dimensional vector space of all vectors $w=\left(w(x): x \in \Omega_{i}\right)$.

Then the formulation (6.18) is equivalent to the linear system of algebraic equations

$$
L_{i} u_{i}=f_{i},
$$

where $u_{i} \in M_{i}$ is the vector of unknowns with components $u_{i}(y), y \in \Omega_{i} ; f_{i} \in M_{i}$ is defined by $f_{i}(x)=\left(f, \varphi_{x}^{i}\right)_{\Omega}$ for all $x \in \Omega_{i} ; L_{i}$ is the matrix whose elements are

$$
L_{i}(x, y)=a\left(\varphi_{y}^{i}, \varphi_{x}^{i}\right), \quad \forall x, y \in \Omega_{i} .
$$

Let us define the usual isomorphism $\mathcal{J}_{i}$ between vectors $v \in M_{i}$ and functions $\tilde{v} \in \mathcal{H}^{i}$ that are their prolongations, i.e.,

$$
\tilde{v}=\mathcal{J}_{i} v \quad \text { means } \quad \tilde{v}(x)=\sum_{y \in \Omega_{i}} v(y) \varphi_{y}^{i}(x), \quad \forall x \in \bar{\Omega},
$$


and, vice versa,

$$
v=\mathcal{J}_{i}^{-1} \tilde{v} \quad \text { is defined by } \quad v(y)=\tilde{v}(y), \quad \forall y \in \Omega_{i} .
$$

Now we introduce the energy norm for functions belonging to $H_{0}^{1}(\Omega)$ :

$$
\|\mid \tilde{v}\|_{\Omega}=\mathcal{L}(\tilde{v}, \tilde{v})^{1 / 2}
$$

and specify the inner product and the norm for vectors in $M_{i}$ :

$$
\begin{aligned}
& (v, w)_{i}=\sum_{x \in \Omega_{i}} v(x) w(x), \\
& \|v\|_{i}=(v, v)_{i}^{1 / 2}, \quad \forall v, w \in M_{i} .
\end{aligned}
$$

From (6.20) and 6.21), we have for an isomorphic pair $v \in M_{i}$ and $\tilde{v}=\mathcal{J}_{i} v \in \mathcal{H}^{i}$ the relationship

$$
\|\mid\| v\left\|_{i}=\right\| \tilde{v} \|_{\Omega}
$$

Now let us introduce the interpolation operator $I_{i}: M_{i} \rightarrow M_{i+1}$. Let $v \in M_{i}$. Since its prolongation $\tilde{v}$ belongs to $\mathcal{H}^{i+1}$, the isomorphism associates with $\tilde{v}$ a vector $w \in M_{i+1}$. In such a way, we have uniquely defined $I_{i}: v \rightarrow w$.

The convergence of the Bubnov-Galerkin solution to the exact solution was studied in a number of papers (e.g., [3]). A standard analysis on a quasi-uniform triangulation gives a non-optimal convergence rate because of a loss of regularity in the exact solution $u$. In our case we can however use the special nested triangulations that are refined towards the singular point and hence obtain the optimal first-order convergence.

Lemma 6.2. Let the triangulations $\mathcal{F}_{i}$ at the levels $i=1, \cdots, l$ be generated with a refinement index

$$
\rho>1 / \mu
$$

and let $\beta=(\rho-1) / \rho$. Suppose that the assumptions (5.3) are satisfied and that $f \in H^{0, \beta}(\Omega)$. Then the solution $\tilde{u}_{i}$ of the Galerkin problem (6.18) satisfies the error estimate

$$
\left\|u-\tilde{u}_{i}\right\|_{\Omega} \leq c_{4} h_{i}\|f\|_{0, \beta} .
$$

Proof. Let us note that $\beta$ satisfies $1-\mu<\beta<1$. This means that we can apply Theorem 5.1 and prove existence of a solution of the continuous problem in $H^{2, \beta}(\Omega)$. Using Lemma 4.5 from [1], we can estimate the interpolation error by

$$
\left\|u-\tilde{v}_{i}\right\|_{1, \Omega} \leq c h_{i}\|u\|_{2, \beta} .
$$

Here $\tilde{v}_{i}$ denotes the piecewise linear interpolant of $u$ on $\mathcal{F}_{i}$. Next, applying (5.12) and using the equivalence of the norms $\||\cdot|\|_{\Omega}$ and $\|\cdot\|_{1, \Omega}$, we get

$$
\left\|u-\tilde{v}_{i}\right\|_{\Omega} \leq c_{4} h_{i}\|f\|_{0, \beta} .
$$

Finally, the optimality of the Galerkin solution $\tilde{u}_{i}$ implies that

$$
\left\|u-\tilde{u}_{i}\right\|_{\Omega} \leq\|\| u-\tilde{v}_{i}\left\|_{\Omega} \leq c_{4} h_{i}\right\| f \|_{0, \beta} .
$$

In the following we need 
Lemma 6.3. Under the assumptions of Lemma 6.2, the solutions $\tilde{u}_{i-1}, \tilde{u}_{i}$ of the Galerkin problem (6.18) in $\mathcal{H}^{i-1}$ and $\mathcal{H}^{i}$ satisfy the inequalities

$$
\begin{aligned}
& \left\|\tilde{u}_{i}-\tilde{u}_{i-1}\right\|_{0,-\beta} \leq c_{5} h_{i}||\left|\tilde{u}_{i}-\tilde{u}_{i-1}\right| \|_{\Omega}, \\
& \quad \| \tilde{u}_{i}-\left.\tilde{u}_{i-1}||\right|_{\Omega} \leq||\left|u-\tilde{u}_{i-1}\right|||_{\Omega} .
\end{aligned}
$$

Proof. We shall use a duality argument as in 11. Let $z$ denote the solution of the problem

$$
a(z, v)=(g, v)_{\Omega} \quad \forall v \in H_{0}^{1}(\Omega)
$$

with the right-hand side

$$
g=\Phi_{\beta}^{-2}\left(\tilde{u}_{i}-\tilde{u}_{i-1}\right) .
$$

We show that $g \in H^{0, \beta}$. From [1] we know that there is a constant $c$ such that for all $v \in H^{1}(\Omega)$ we have

$$
\|v\|_{0,-\beta} \leq c\|v\|_{1} .
$$

Setting $v=\tilde{u}_{i}-\tilde{u}_{i-1}$, we get

$$
\|g\|_{0, \beta}=\left\|\tilde{u}_{i}-\tilde{u}_{i-1}\right\|_{0,-\beta} \leq\left\|\tilde{u}_{i}-\tilde{u}_{i-1}\right\|_{1}<\infty .
$$

Let $\tilde{z}_{i-1}$ be the Galerkin solution of the problem

$$
a\left(\tilde{z}_{i-1}, v\right)=(g, v)_{\Omega} \quad \forall v \in \mathcal{H}^{i-1} .
$$

Applying Lemma 6.2, we obtain

$$
\left\|\mid z-\tilde{z}_{i-1}\right\|_{\Omega} \leq c_{4} h_{i-1}\left\|\tilde{u}_{i}-\tilde{u}_{i-1}\right\|_{0,-\beta} .
$$

From (6.30) and (6.18) we get the representation

$$
\left\|\tilde{u}_{i}-\tilde{u}_{i-1}\right\|_{0,-\beta}^{2}=a\left(z-\tilde{z}_{i-1}, \tilde{u}_{i}-\tilde{u}_{i-1}\right) .
$$

By means of the Cauchy-Bunjakovski inequality we obtain

$$
\begin{aligned}
\left\|\tilde{u}_{i}-\tilde{u}_{i-1}\right\|_{0,-\beta}^{2} & \leq\left|\left\|z-\tilde{z}_{i-1}\left|\left\|\left.\right|_{\Omega}\right\|\right| \tilde{u}_{i}-\tilde{u}_{i-1}\right\|\right|_{\Omega} \\
& \leq c_{4} h_{i-1}\left\|\tilde{u}_{i}-\tilde{u}_{i-1}\right\|_{0,-\beta}\left\|\mid \tilde{u}_{i}-\tilde{u}_{i-1}\right\| \|_{\Omega},
\end{aligned}
$$

which yields (6.28) with $c_{5}=2 c_{4}$.

In order to prove (6.29), we set $v=\tilde{u}_{i}-\tilde{u}_{i-1}$ in (5.4) and (6.18); this gives

$$
a\left(u, \tilde{u}_{i}-\tilde{u}_{i-1}\right)=a\left(\tilde{u}_{i}, \tilde{u}_{i}-\tilde{u}_{i-1}\right),
$$

and taking into consideration

$$
a\left(\tilde{u}_{i-1}, \tilde{u}_{i}-\tilde{u}_{i-1}\right)=0,
$$

we obtain the representation

$$
a\left(u-\tilde{u}_{i}, \tilde{u}_{i}-\tilde{u}_{i-1}\right)=a\left(\tilde{u}_{i}-\tilde{u}_{i}, \tilde{u}_{i}-\tilde{u}_{i-1}\right) .
$$

Estimating the left-hand side by the Cauchy-Bunjakovski inequality,

$$
\begin{aligned}
\left\|\tilde{u}_{i}-\tilde{u}_{i-1} \mid\right\|_{\Omega}^{2} & =a\left(u-\tilde{u}_{i-1}, \tilde{u}_{i}-\tilde{u}_{i-1}\right) \\
& \leq|| u-\left.\tilde{u}_{i-1}||\right|_{\Omega}||\left|\tilde{u}_{i}-\tilde{u}_{i-1}\right| \|_{\Omega},
\end{aligned}
$$

we finally get (6.29).

In order to check the convergence criterion (4.1) in the next section, we need a result on the equivalence of norms. 
Lemma 6.4. Let $v \in M_{i}, \tilde{v}=\mathcal{J}_{i} v \in \mathcal{H}^{i}$ be an isomorphic pair, let $\rho \geq 1$ be the refinement index of the triangulation, and $\beta=(\rho-1) / \rho$. Then there are constants $c_{7}, c_{8}>0$, which are independent of $i, v$, and $\tilde{v}$, such that

$$
c_{7}\|\tilde{v}\|_{0,-\beta} \leq h_{i}\|v\|_{i} \leq c_{8}\|\tilde{v}\|_{0,-\beta} .
$$

Proof. First we consider a triangle $\triangle a_{1} a_{2} a_{3} \in \mathcal{F}_{i}$ that does not contain $A=(0,0)$. Then we have (6.3), which gives

$$
L^{-2} \frac{h_{i}^{2}}{d_{\triangle}^{2}} \int_{\triangle} \tilde{v}^{2} d x \leq \int_{\triangle} \tilde{v}^{2} \Phi_{\beta}^{-2} d x \leq L^{2} \frac{h_{i}^{2}}{d_{\triangle}^{2}} \int_{\triangle} \tilde{v}^{2} d x .
$$

From (6.2), we have the affine regularity of $\triangle$ and get, analogously to Theorem 3.13 of [13],

$$
\begin{gathered}
c_{9}^{-1} d_{\triangle}^{2}\left(v^{2}\left(a_{1}\right)+v^{2}\left(a_{2}\right)+v^{2}\left(a_{3}\right)\right) \leq \int_{\triangle} \tilde{v}^{2} d x \\
\leq c_{9} d_{\triangle}^{2}\left(v^{2}\left(a_{1}\right)+v^{2}\left(a_{2}\right)+v^{2}\left(a_{3}\right)\right),
\end{gathered}
$$

with a constant $c_{9}$ that is independent of $\triangle$. Thus

$$
\begin{gathered}
c_{9}^{-1} L^{-2} h_{i}^{2}\left(v^{2}\left(a_{1}\right)+v^{2}\left(a_{2}\right)+v^{2}\left(a_{3}\right)\right) \leq \int_{\triangle} \tilde{v}^{2} \Phi_{\beta}^{-2} d x \\
\leq c_{9} L^{2} h_{i}^{2}\left(v^{2}\left(a_{1}\right)+v^{2}\left(a_{2}\right)+v^{2}\left(a_{3}\right)\right) .
\end{gathered}
$$

Second, we consider a triangle $\triangle \in \mathcal{F}_{i}$ that contains $A=(0,0)$, e.g., $\triangle A B_{i} C_{i}$. From the left-hand side of (6.4), we get

$$
L^{-1} \Phi_{\beta}(x) \leq d_{\triangle} / h_{i} \quad \forall x \in \triangle .
$$

Therefore we obtain as above the left-hand side of (6.36) for this $\triangle$. It remains to show the second inequality of (6.36). For this we introduce barycentric coordinates $\lambda_{1}(x), \lambda_{2}(x), \lambda_{3}(x)$ corresponding to the vertices $B_{i}, C_{i}, A$ respectively. Since $v(A)=0$, we have

$$
\tilde{v}(x)=v\left(B_{i}\right) \lambda_{1}(x)+v\left(C_{i}\right) \lambda_{2}(x) \quad \forall x \in \triangle .
$$

Let $C_{i}=\left(b_{1}, b_{2}\right)$. Then

$$
\left|\lambda_{1}(x)\right|=\left|x_{1} b_{2}-x_{2} b_{1}\right| /(2 \text { meas } \triangle) .
$$

From the Cauchy-Bunjakovski inequality, we obtain

$$
\left|\lambda_{1}(x)\right| \leq r(x) d_{\triangle} /(2 \text { meas } \triangle) \quad \forall x \in \triangle .
$$

The Law of Sines and (6.2) imply that

$$
2 \text { meas } \triangle \geq d_{\triangle}^{2} \sin ^{2}(1 / L) \text {. }
$$

Consequently we have

$$
\left|\lambda_{1}(x)\right| \leq r(x) /\left(d_{\triangle} \sin ^{2}(1 / L)\right) .
$$

Analogously, we get

$$
\left|\lambda_{2}(x)\right| \leq r(x) /\left(d_{\triangle} \sin ^{2}(1 / L)\right) .
$$

From the second inequality of (6.4), we have

$$
d_{\triangle} / h_{i} \leq L \sup _{\triangle} r^{\beta}(x) \leq L d_{\triangle}^{\beta}, \quad \text { i.e., } \quad d_{\triangle}^{1-\beta} \leq L h_{i} .
$$


Using (6.37)-(6.39), we get

$$
\begin{aligned}
\int_{\triangle} \tilde{v}^{2}(x) r^{-2 \beta}(x) d x & =\int_{\triangle} r^{-2 \beta}(x)\left(v\left(B_{i}\right) \lambda_{1}(x)+v\left(C_{i}\right) \lambda_{2}(x)\right)^{2} d x \\
& \leq c_{10} d_{\triangle}^{-2}\left(\left|v\left(B_{i}\right)\right|+\left|v\left(C_{i}\right)\right|\right)^{2} \int_{\triangle} r^{2-2 \beta}(x) d x \\
& \leq 2 c_{10} d_{\triangle}^{-2}\left(v^{2}\left(B_{i}\right)+v^{2}\left(C_{i}\right)\right) \int_{0}^{\theta} \int_{0}^{d_{\triangle}} r^{3-2 \beta} d r d \varphi \\
& \leq c_{10} \theta d_{\triangle}^{2-2 \beta}\left(v^{2}\left(B_{i}\right)+v^{2}\left(C_{i}\right)\right) \\
& \leq c_{10} \theta L^{2} h_{i}^{2}\left(v^{2}\left(B_{i}\right)+v^{2}\left(C_{i}\right)\right),
\end{aligned}
$$

where $c_{10}=\sin ^{-2}(1 / L)$. Now we are able to prove the second inequality of (6.34) by summing the left-hand side of (6.36) over all triangles $\triangle \in \mathcal{F}_{i}$ :

$$
c_{9}^{-1} L^{-2} h_{i}^{2}\|v\|_{i}^{2}=c_{9}^{-1} L^{-2} h_{i}^{2} \sum_{y \in \Omega_{i}} v^{2}(y) \leq \int_{\Omega} \tilde{v}^{2} \Phi_{\beta}^{-2} d x=\|\tilde{v}\|_{0,-\beta}^{2} .
$$

Thus we can take $c_{8}=\sqrt{c_{9}} L$ in (6.34). To prove the first inequality of (6.34), we sum the second inequality of (6.36) over all triangles $\Delta \in \mathcal{F}_{i}$ that do not contain $A$ and (6.41) over all triangles $\triangle \in \mathcal{F}_{i}$ that do contain $A$. This gives

$$
\|\tilde{v}\|_{0,-\beta}^{2}=\int_{\Omega} \tilde{v}^{2}(x) r^{-2 \beta}(x) d x \leq c_{11} k L^{2} h_{i}^{2} \sum_{y \in \Omega_{i}} v^{2}(y)=c_{11} k L^{2} h_{i}^{2}\|v\|_{i}^{2},
$$

where $c_{11}=\max \left\{c_{9}, c_{10} \theta\right\}$ and $k$ is the maximum number of triangles having a common vertex. Therefore we can take $c_{7}=c_{11}^{-1 / 2} k^{-1 / 2} L^{-1}$ in (6.34).

\section{The MAIn CONVERGENCE RESUlt}

Now we are ready to apply the abstract convergence result of Theorem 4.1 to the boundary value problem.

Theorem 7.1. Let the assumptions (5.3) and (5.13) on the data be satisfied and let the refinement index $\rho$ of the triangulation be greater than $1 / \mu$. Then for the $C C G$-algorithm with $m_{j}$ iterations on each level $j$ for $j=1, \cdots, l$, we have the error estimate

$$
||\left|u_{l}-v_{l}\right|\left\|_{l}=|| \mid \tilde{u}_{l}-\tilde{v}_{l}\right\|_{\Omega_{\Omega}} \leq c_{12} \sum_{j=1}^{l} \frac{h_{j}}{2 m_{j}+1}\|f\|_{0},
$$

where $\tilde{v}_{l}=\mathcal{J}_{l} v_{l}$ and $v_{l}$ denotes the final approximation of the CCG-algorithm.

Proof. Let us note that for $i=1, \cdots, l$ we have the usual estimate

$$
0<\lambda_{i}^{*} \leq c_{6}
$$

for the largest eigenvalue $\lambda_{i}^{*}$ of the matrix $L_{i}$ (see, e.g., [13, Theorem 3.14]). Taking into consideration (6.28) and (6.23), we get

$$
\left\|\tilde{u}_{i}-\tilde{u}_{i-1}\right\|_{0,-\beta} \leq c_{5} h_{i} \mid\left\|u_{i}-I_{i-1} u_{i-1}\right\|_{i} .
$$

The norm equivalence (6.34) gives

$$
h_{i}\left\|u_{i}-I_{i-1} u_{i-1}\right\|_{i} \leq c_{8}\left\|\tilde{u}_{i}-\tilde{u}_{i-1}\right\|_{0,-\beta} .
$$


Combining these inequalities with (7.2), we obtain

$$
\left\|u_{i}-I_{i-1} u_{i-1}\right\|_{i} \leq c_{5} c_{8} \sqrt{\frac{c_{6}}{\lambda_{i}^{*}}}\left\|\left|u_{i}-I_{i-1} u_{i-1}\right|\right\|_{i} .
$$

This proves (4.1) for $\gamma=1$ and $c^{*}=c_{5} c_{8} \sqrt{c_{6}}$. Since all the assumptions of Theorem 4.1 are satisfied, we get

$$
\left\|\left|u_{l}-v_{l}\left\|_{l} \leq c^{*} \sum_{j=1}^{l} \frac{1}{2 m_{j}+1}\right\|\right| \tilde{u}_{j}-\tilde{u}_{j-1}\right\|_{\Omega}
$$

from (3.9) and (6.23). Owing to (6.29) and (6.25), we have (7.1) with $c_{12}=c^{*} c_{2} c_{4}$.

Now we count the number $N_{C C G}$ of arithmetic operations for the full CCGalgorithm and try to choose $m_{j}$ in an optimal way. Upper bounds for the arithmetic operations have been considered, e.g., in [12], [13], and are given by

$$
N_{C C G} \leq N\left(m_{1}, \cdots, m_{l}\right):=d_{1} \sum_{j=1}^{l}\left(m_{j}+d_{2}\right) n_{j}+d_{3}
$$

with constants $d_{1}, d_{2}$ and $d_{3}$ that are independent of $m_{j}, n_{j}$ and $h_{j}$. On the other hand, the accuracy of the CCG-algorithm is characterized by the value $\varepsilon=$ $\varepsilon\left(m_{1}, \ldots, m_{l}\right)$ :

$$
\left\|\tilde{u}_{l}-\tilde{v}_{l}\right\|_{\Omega} \leq \varepsilon\left(m_{1}, \cdots, m_{l}\right):=d_{4} \sum_{j=1}^{l} \frac{h_{j}}{2 m_{j}+1}
$$

with the constant $d_{4}=c_{12}\|f\|_{0}$. This leads us to solve the optimization problem:

Find $m_{1}, \cdots, m_{l}$ such that the value $\varepsilon\left(m_{1}, \cdots, m_{l}\right)$ is minimal under the constraint

$$
N\left(m_{1}, \cdots, m_{l}\right) \leq d_{5}
$$

for an appropriate constant $d_{5}>0$.

Applying the method of Lagrange multipliers, we obtain

$$
\left(2 m_{j}+1\right)^{2}=d_{6} h_{j} / n_{j}, \quad \text { for } j=1, \ldots, l,
$$

with a constant $d_{6}$ that depends on $d_{1}, \cdots, d_{5}$ but is independent of $m_{j}$. We eliminate $d_{6}$ by using (7.9) for $j=l$, and get

$$
2 m_{j}+1=\left(2 m_{l}+1\right) \sqrt{h_{j} n_{l} / h_{l} n_{j}} .
$$

Since $h_{j}=2^{l-j} h_{l}$ and $n_{l} \approx 4^{l-j} n_{j}$, we obtain

$$
2 m_{j}+1 \approx\left(2 m_{l}+1\right) 2^{3(l-j) / 2}
$$

We use this relation in the following way. Fixing the number of iterations at the highest level $l$ by setting $m_{l}=m$, we choose $m_{j}$ at the lower levels as the smallest integer $m_{j}$ such that

$$
2 m_{j}+1 \geq\left(2 m_{l}+1\right) 2^{3(l-j) / 2}, \quad \text { for } \quad j=1, \cdots, l-1 .
$$


Theorem 7.2. In addition to the assumptions of Theorem 7.1, let the number $m_{j}$ of iterations in the CCG-algorithm be defined by (7.12). Then the error of the final approximation $v_{l}$ in the CCG-algorithm satisfies the estimates

$$
\left\|\left|u_{l}-v_{l}\left\|_{l}=\right\|\right| \tilde{u}_{l}-\tilde{v}_{l}\right\|_{\Omega} \leq c_{13} \frac{h_{l}}{2 m+1}\|f\|_{0}
$$

and

$$
\left.\left\|u-\tilde{v}_{l}\right\|\right|_{\Omega} \leq h_{l}\left(c_{2} c_{4}+\frac{c_{13}}{2 m+1}\right)\|f\|_{0},
$$

where $\tilde{v}_{l}=\mathcal{J}_{l} v_{l}$ and the constants $c_{2}$ and $c_{4}$ are given in (5.14) and (6.25). The number of arithmetic operations is bounded by

$$
N_{C C G} \leq\left(c_{14} m+c_{15}\right) n_{l}
$$

the constants $c_{13}-c_{15}$ are independent of the number of levels, the number of $C G$ iterations on the highest level $m$, and the number of unknowns on the highest level $n_{l}$.

Proof. Using (17.12) and the relation $h_{j}=2^{l-j} h_{l}$ in (17.1), we get

$$
\left\|\mid \tilde{u}_{l}-\tilde{v}_{l}\right\|_{\Omega} \leq c_{12} \frac{h_{l}}{2 m+1} \sum_{j=1}^{l} 2^{-(l-j) / 2}\|f\|_{0} .
$$

Summing, we get (7.13) with the constant $c_{13}=c_{12} /\left(1-2^{-1 / 2}\right)$. From the Euler formula for a polyhedron, we have

$$
n_{l} \geq 4^{l-j} n_{j}
$$

The definition of $m_{j}$ implies that

$$
2 m_{j}+1 \leq(2 m+1) 2^{3(l-j) / 2}+2 .
$$

Using these inequalities in (7.6), we obtain the estimate

$$
N_{C C G} \leq d_{1} n_{l} \sum_{j=1}^{l}\left((2 m+1) 2^{3(l-j) / 2}-1 / 2+d_{2}\right) 2^{-2(l-j)}+d_{3} .
$$

Calculating the sum, we finally get (7.15) with constants

$$
c_{14}=2 /\left(1-2^{-1 / 2}\right) \quad \text { and } \quad c_{15}=1 /\left(1-2^{-1 / 2}\right)+\left(4 d_{2}-2\right) / 3+d_{3} .
$$

Remark 7.3. Now choosing $m$ in order to get $c_{2} c_{4} \sim c_{13} /(2 m+1)$, we see that the amount of arithmetic operations is proportional only to $n_{l}$.

Remark 7.4. The inequality (7.14) shows that the final numerical solution produced by the CCG-method is, in the energy norm, of the same order of magnitude as the discretization error of the finite element method. Nevertheless, this approximate solution $\tilde{v}_{l}$ is not the finite element solution and may not have a higher-order $L^{2}$ norm error nor exhibit superconvergence. In this sense, the CCG-method is not as good as multigrid V-cycle iterations or the CG-method with a V-cycle preconditioner [13], [14].

Remark 7.5. The inequality (7.12) gives an upper bound for the estimated number of iterations on each level. For the coarse grids this bound may be too pessimistic (i.e., much larger than the number of unknowns). 


\section{ACKNOWLEDGMENTS}

The authors acknowledge Prof. J. Xu for the discussion that motivated these investigations. Moreover, the authors wish to thank the German Research Association (DFG) for supporting the research presented in this paper.

\section{REFERENCES}

1. I. Babuška, R.B. Kellogg, and J. Pitkäranta, Direct and inverse error estimates for finite elements with mesh refinements, Numer. Math. 33 (1979), 447-471. MR 81c:65054

2. F.A. Bornemann, On the convergence of cascadic iterations for elliptic problems, Tech. Report SC 94-8, Konrad-Zuse-Zentrum Berlin (ZIB), 1994.

3. P. Ciarlet, The Finite Element Method for Elliptic Problems, North-Holland, Amsterdam, 1978. MR 58:25001

4. P. Deuflhard, Cascadic Conjugate Gradient Methods for Elliptic Partial Differential Equations I. Algorithm and Numerical Results, Tech. Report SC 93-23, Konrad-Zuse-Zentrum Berlin (ZIB), 1993.

5. _ Cascadic conjugate gradient methods for elliptic partial differential equations. Algorithm and numerical results, Domain decomposition methods in scientific and engineering computing. Proceedings of the 7th international conference on domain decomposition, October 27-30, 1993, Pennsylvania State University, (D. Keyes and J. Xu, eds.), Contemp. Math., vol. 180, Providence, RI: American Mathematical Society, 1994, pp. 29-42. MR 95i:65008

6. P. Grisvard, Singularities in Boundary Value Problems, Springer-Verlag, Berlin, and Masson, Paris, 1992. MR 93h:35004

7. V.A. Kondrat'ev, Boundary problems for elliptic equations in domains with conical or angular points, Trans. Mosc. Math. Soc. 16 (1967), 227-313. MR 37:1777

8. O.A. Ladyzhenskaya and N.N. Uraltseva, Linear and quasilinear elliptic equations, Academic Press, New York, 1968. MR 39:5941

9. L.A. Oganesjan and L.A. Rukhovets, Variational Difference Methods of Solving the Elliptic Equations (in Russian), Acad. Sci. of the Armenian SSR, Erevan, 1979. MR 92m:65101

10. A.A. Samarskii and E.S. Nikolaev, Numerical Methods for Grid Equations. Vol. II, Iterative Methods, Birkhäuser, Basel, 1989. MR 90m:65003

11. V.V. Shaidurov, The convergence of the cascadic conjugate-gradient method under a deficient regularity, Problems and Methods in Mathematical Physics (L.Jentsch and F.Tröltzsch, eds.), Teubner, Stuttgart, 1994, 185-194. MR 95f:65217

12. Some estimates of the rate of convergence for the cascadic conjugate-gradient method, Comput. Math. Appl. 31 (1996), No. 4-5, 161-171. MR 96j:65003

13. MR 97e:65142

14. H. Yserentant, The convergence of multi-level methods for solving finite-element equations in the presence of singularities, Math. Comp. 47 (1986), 399-409. MR 88d:65149

Institute of Computational Modelling, Siberian Branch of the Russian Academy of SCIENCES, KRASNOYARSK 660036, Russia

E-mail address: shidurov@cckr.krasnoyarsk.su

Otto-von-Guericke-Universität Magdeburg, Postfach 4120, D-39016 Magdeburg, Germany

E-mail address: tobiska@mathematik.uni-magdeburg.de 